\title{
Integrating community health representatives with health care systems: clinical outcomes among individuals with diabetes in Navajo Nation
}

Letizia Trevisi ${ }^{1}$, John E. Orav², Sidney Atwood ${ }^{3}$, Christian Brown² ${ }^{2}$ Cameron Curley ${ }^{3}$, Caroline King ${ }^{4}$, Olivia Muskett ${ }^{3}$, Hannah Sehn ${ }^{5}$, Katrina A. Nelson ${ }^{3}$, Mae-Gilene Begay ${ }^{6}$ and Sonya S. Shin ${ }^{3^{*}}$ (i)

\begin{abstract}
Background: We studied the impact of Community Outreach and Patient Empowerment (COPE) intervention to support Community Health Representatives (CHR) on the clinical outcomes of patients living with diabetes in the Navajo Nation extending into the States of Arizona, Utah, and New Mexico. The COPE intervention integrated CHRs into healthcare teams by providing a structured approach to referrals and home visits.

Methods: We abstracted routine clinical data from the Indian Health Service's information system on individuals with diabetes mellitus seen at participating clinical sites from 2010 to 2014. We matched 173 COPE participants to 2880 patients with similar demographic and clinical characteristics who had not participated in COPE. We compared the changes in clinical outcomes between the two groups using linear mixed models.

Results: Over the four years of the study, COPE patients had greater improvements in glycosylated hemoglobin ( $-0.56 \%)$ than non-COPE participants (+ 0.07\%) for a difference in differences of $0.63 \%$ (95\% confidence interval (Cl): 0.50, 0.76). Lowdensity lipoprotein fell more steeply in the COPE group $(-10.58 \mathrm{mg} / \mathrm{dl})$ compared to the non-COPE group $(-3.18 \mathrm{mg} / \mathrm{dl})$ for a difference in differences of $7.40 \mathrm{mg} / \mathrm{dl}(95 \% \mathrm{Cl}: 2.00,12.80)$. Systolic blood pressure increased slightly more among COPE $(2.06 \mathrm{mmHg})$ than non-COPE patients $(0.61 \mathrm{mmHg})$. We noted no significant change for body mass index in either group.
\end{abstract}

Conclusion: Structured outreach by Community Health Representatives as part of an integrated care team was associated with improved glycemic and lipid levels in the target Navajo population.

Trial registration: Trial registration: NCT03326206. Registered 31 October 2017 - Retrospectively registered, https://clinicaltrials.gov/ct2/show/study/NCT03326206.

Keywords: Chronic disease, Community health workers, Diabetes mellitus, Education and behavioral intervention, Navajo

\section{Background}

Diabetes mellitus (DM) is a prevalent chronic condition in many Native communities. Cardiovascular disease and type $2 \mathrm{DM}$ (T2DM) are the 3rd and 4th leading causes of death in the Navajo Nation, respectively [1]. Mortality due to diabetes is almost twice as high among American Indian and Alaska Native populations than in

\footnotetext{
* Correspondence: sshin@bwh.harvard.edu

${ }^{3}$ Division of Global Health Equity, Brigham and Women's Hospital, Boston, MA, USA

Full list of author information is available at the end of the article
}

the general population in the United States [2]. The cost of T2DM care among Native populations is considerable, estimated in one Indian Health Service (IHS) facility to account for $37 \%$ of all adult treatment expenses [3]. Lack of access to healthcare services and healthy food contribute significantly to the burden of diabetes [4] as well as other chronic health disparities affecting rural Native communities. Some scholars have argued that the rising rate of T2DM over the past few decades in Native communities correlates with the historical imposition of U.S. federal policies that have eroded local 
indigenous food systems and traditional lifestyles and diets [5].

Despite these structural challenges, unique strengths exist within tribal health care systems. One such resource is the Community Health Representative (CHR) Program which is instrumental in supporting and promoting health by addressing the social determinants of health at the community level. The CHR is a national cadre of welltrained, medically-guided, Native community-based health care providers who may include traditional Native concepts in their work [6].

The Community Outreach and Patient Empowerment (COPE) project was launched in 2010 in collaboration with the Navajo Nation CHR Program to improve health outcomes by supporting CHR outreach to individuals living with chronic diseases. The purpose of the intervention is to improve management of T2DM and comorbidities in order to reduce future cardiovascular risk $[7,8]$. As a programmatic intervention, COPE focuses on increasing referrals of high-risk patients to Navajo Nation CHRs, supporting CHRs through training and standardized health promotion materials, and strengthening care coordination between CHRs and clinic-based providers. To date, this program has enrolled more than 650 patients in the Navajo Nation.

The purpose of this study is to measure the impact of the COPE program on the clinical outcomes of individuals living with T2DM in the Navajo Nation, who were referred to the COPE program by providers or CHRs themselves. The main outcome assessed is the change in glycosylated hemoglobin (HbA1c) averaged over the 24 months prior to enrollment in COPE and the 24 months after enrollment.

\section{Methods}

\section{Study setting and population}

The Navajo Nation includes 332,129 members and covers 27,400 square miles $\left(71,000 \mathrm{~km}^{2}\right)$ in parts of Arizona, Utah, and New Mexico [9]. It is organized into 110 Chapters, which are local government entities similar to counties or townships. The healthcare services on Navajo Nation are delivered in two different ways: through the Navajo Area Indian Health Service, one of 12 regional administrative units of the national IHS system, and through tribally-contracted health programs. The Navajo Area Indian Health Service operates in 5 hospitals, 7 health centers, and 15 clinics. The majority of population served by the Navajo Area IHS lives on the rural reservation. Along with its vast geography and limited infrastructure (with $78 \%$ of public roads still unpaved), low incomes and low population density discourage retail food sources on the reservation. Currently, an estimated 25,000 Navajo individuals are living with T2DM, and another 75,000 have pre-DM, encompassing $49.6 \%$ of the adult population
[10]. The paucity of health facilities and high turnover of healthcare workers, combined with efforts to incorporate public health services into tribal healthcare, led to the introduction of the CHR program.

\section{Role of CHRs in Navajo Nation}

The Navajo Nation employs nearly 100 CHRs to connect health facilities and medical providers to communities and help community members navigate these complex health systems by facilitating access to healthcare services, improving the quality of healthcare delivery, and offering community-based outreach for patients, families, and communities. CHRs are trained as Certified Nursing Assistants and are required to speak Navajo. Each CHR is assigned to one or more specific Chapter, depending on the size of the Chapters, vacancies, and the number of clients who need close monitoring. CHRs cover an average number of two Chapters, and have a caseload that ranges from 30 to 50 clients. While CHRs are available to everyone in their communities, they prioritize outreach to clients with chronic health conditions (e.g. T2DM, heart disease) and/or risk factors associated with social determinants of health based on their assessment of each client's social support and living conditions. CHRs conduct home visits to these clients to check vital signs, assess medication adherence, monitor for any urgent health issues, and answer any questions that the patient might have. As Certified Nursing Assistants, CHRs are qualified to monitor vital signs and measure blood glucose levels using finger stick testing. CHRs also assist with many social issues by referring clients to programs that improve unsafe housing (e.g. adding a bathroom if there's only an outhouse, wheelchair ramp), referring clients to food assistance programs, and coaching patients on how to arrange transportation to medical appointments.

\section{Program intervention}

COPE is a multi-level intervention aimed at linking clinic-based providers, $\mathrm{CHRs}$, and patients. The program is comprised of three inter-related strategies designed to strengthen the existing community outreach and linkage to clinic-based care [11].

\section{1) Enabling patient referral to the Navajo Nation} Community Health Representative Program. Providers are asked to refer patients at increased risk of chronic disease complications. The provider discusses enrollment with the patient and if the patient agrees, complete a referral to the CHR program, either through the IHS electronic health record or using a standard IHS paper referral form. CHRs may also refer clients identified through home visits, and clients may self-refer (or be 
referred by their family members) to the program (Table 1). Because CHRs provide services exclusively to individuals living on the reservation, patients enrolled in the program typically face significant geographic barriers to access healthcare and healthy food, and limited infrastructure such as utilities and paved roads [Table 1].

2) Supporting community-based patient accompaniment. The COPE team routinely provides additional training to CHRs in evidence-based behavior change strategies, such as Motivational Interviewing, patient-identified SMART goals (i.e. Specific, Measurable, Attainable, Relevant, Time bound), and delivers structured teaching materials in the form of flipcharts. COPE has developed culturally-informed flipcharts to cover specific health topics (e.g. cholesterol, diabetes foot care, physical activity) following the format of the 5A (Ask, Assess, Advise, Assist, Arrange follow-up) brief counseling intervention $[12,13]$. Upon referral, CHRs make regular home visits to COPE participants, generally once or twice a month.
3) Community-clinical linkages. The COPE team works with CHRs and providers to increase bidirectional communication about patient management. COPE team works with providers to create access for CHRs on Electronic Health Records, initiate case management, create templates for electronic CHR referrals, and orient providers on CHRs' work and the COPE intervention.

All program participants receive routine standard of care in addition to the COPE intervention.

The COPE team was careful to allow CHRs to have flexibility in how they chose to deliver the intervention to their clients. The components that were consistently delivered to all COPE patients were: 1) health education using flipcharts at least one module monthly, 2) vital signs including blood glucose checks (if patient allowed) at each home visit, 3) communication with provider regarding any urgent or pressing clinical issues. Since the COPE team has developed the flipcharts and works with local providers to deliver training to the CHRs, the cost of the intervention maintenance is minimal, requiring

Table 1 COPE intervention additional components, Navajo Nation, United States, 2010-2014

\begin{tabular}{ll}
\hline $\begin{array}{l}\text { Program } \\
\text { Components }\end{array}$ & Before COPE collaboration \\
\hline $\begin{array}{ll}\text { Patient } \\
\text { outreach }\end{array}$ & Home visits by CHRs without established frequency. \\
& $\begin{array}{l}\text { Each CHR prepares his/her own health education materials resulting in } \\
\text { inconsistent health coaching. }\end{array}$
\end{tabular}

Vital signs monitored inconsistently, CHRs lack oximeters, multiple size blood pressure cuffs, or glucometer training / supplies.

CHR Training CHRs receive training on health topics when available.

CHRs do not receive training on motivational interviewing, self-care, goal setting.

Not competency assessments of CHR or trainer knowledge / proficiency.

CHR supervisors receive training when available.

linkages
CHRs work with Public Health Nurses to evaluate clients together and establish care plans; however, CHRs rarely coordinate care with other healthcare providers.

No access to Electronic Health Records for CHRs.

Patients rarely referred by providers to $\mathrm{CHRs}$; primarily identified by CHRs themselves.
Introduced by COPE collaboration

"COPE clients" receive home visits at least monthly and tracked as high-risk client.

CHRs deliver standardized coaching materials that have been vetted by local providers and ensure goal setting at each session.

Vital signs monitored; all CHRs equipped with oximeters, multiple size blood pressure cuffs, glucometer training / supplies.

Monthly training sessions to CHRs on health topics taught by local providers to build CHR-provider relationship.

CHRs receive training on motivational interviewing, selfcare, goal setting delivered by Navajo-speaking trainers.

Competency assessments administered at each training to assess CHR and trainer knowledge / proficiency.

CHR supervisors receive monthly trainings in team building, supervision and leadership, quality improvement, and wellness / self-care.

Increased bi-directional communication and care coordination through planning conjunct meetings, orientation of new clinical staff, provider-led CHR trainings, joint home visits, and conjunct case management.

CHRs are able to gain access to Electronic Health Records to document home visits and obtain client information.

COPE helped to increase the awareness of the CHR program with presentations in hospitals. Referral system established and increased referrals by providers to CHR Program. 
ongoing time contributed by providers to train and ensure that flipcharts are updated (Table 1).

\section{Study design and outcomes}

This quasi-experimental study was designed to evaluate the impact of the COPE intervention in patients living with T2DM by comparing clinical endpoints among DM patients receiving COPE and a comparable group that did not receive the intervention. Using routine clinical data from the IHS's information system in six federal sub-regions, we identified patients enrolled in the COPE program who had an ICD-10 diagnosis of T2DM and received care at one of the participating study facilities. We abstracted laboratory test results, vital signs, medications, and health utilization data from 2009 to 2015 for these patients from the Resource and Patient Management System (RPMS), an electronic health record used by the majority of IHS facilities for routine clinical care. Of note, sociodemographic characteristics, such as educational level or income, were not available in routine medical records. Next, we identified non-COPE patients and included into the study those that could be matched to individuals in the intervention group on age $( \pm 5$ years), gender, primary health facility, and baseline hemoglobin HbA1c ( \pm 1 point) and systolic blood pressure $( \pm 10 \mathrm{mmHg})$ where baseline refers to a time point three months or less prior to the date of the COPE patient's enrollment in the program. We assessed the difference in differences for the mean hemoglobin HbAlc during the 24 months prior to enrollment in COPE and the 24 months after enrollment. We also assessed changes in low-density lipoprotein (LDL), systolic blood pressure (SBP), and body mass index (BMI) for the subsets of patients with baseline essential hypertension, diagnoses of dyslipidemia, and a body mass index $>30$, respectively.

To adjust for potential confounders, we collected data on the following patient characteristics: age (continuous), gender (male/female), preferred language (English, Navajo and other), whether the patient had a primary care physician; essential hypertension, major depression disorder, alcohol abuse, dyslipidemia, and major cardiovascular disease. We considered a participant to have major cardiovascular disease if he/she had one of the following diagnoses: acute myocardial infarction, coronary artery bypass surgery, coronary angioplasty, peripheral arterial disease, abdominal aortic aneurysm, carotid artery disease, cerebrovascular disease. We assessed the quality of clinical monitoring in COPE and non-COPE patients using a binary indicator that indicated whether HbA1c, LDL, and SBP had all been measured at least once between 12 and 24 months after enrollment. For this analysis we included all individuals with a preenrollment HbA1c value up to 24 months prior to enrollment, generating matched non-COPE to COPE participants using the same baseline variables described above.

\section{Statistical approach}

We used mixed linear regression models to assess differences over time in the continuous outcome values and logistic regression to assess clinical monitoring, adjusting for the baseline outcome as averaged over the 2 years preceding the enrollment date and including a random effect term for patient and facility (Additional file $1 \mathrm{Ap}$ pendix). We also included a random effect term for each matching set of COPE and non-COPE patients. We performed sensitivity analyses adjusting for the health facility effect as a fixed rather than random effect, and we evaluated the clinical outcomes using a narrower timewindow: 12 months pre- and 12 months post-enrollment period (rather 24 months). All the analyses were carried out using SAS 9.3 (SAS Institute, Cary NC).

\section{Study registration}

Our study design is observational and does not involve prospective assignment to an intervention. Patients were referred or not referred to COPE outreach by their provider or CHRs themselves as part of routine care, before the observational study began. For this reason, we do not consider this study a clinical trial. However, PCORI requires all studies funded by their organization must be registered in a study database; based on researching the different study registries, we felt that clinicaltrials.gov would be the most suitable. For this reason, the study was registered in clinicaltrials.gov after enrollment began.

\section{Results}

\section{Study cohort and baseline characteristics}

From December 2010 to August 2014, we identified 173 individuals COPE clients and 2880 non-COPE patients who met our inclusion criteria. The ratio of COPE to non-COPE participants varied depending on the number of matches; the median number of non-COPE patients matched to each COPE patient was 11 [range: 1-85].

As shown in Table 2, COPE and non-COPE patients were similar in most of patient characteristics and clinical diagnoses, except for primary language, and dyslipidemia. Among COPE clients, 58\% reported Native American as their primary language compared to $41 \%$ of the non-COPE group. The prevalence of dyslipidemia at baseline was $46 \%$ among COPE patients and 59\% among non-COPE patients. [Table 2] The baseline HbA1c LSM was $8.39 \%$ among COPE patients, and $8.23 \%$ in the comparison group, the baseline LDL LSM among COPE was $95.70 \mathrm{mg} / \mathrm{dl}$ versus $95.52 \mathrm{mg} / \mathrm{dl}$ of non-COPE, the baseline SBP LSM was $132.46 \mathrm{mmHg}$ in the intervention 
group and $131.45 \mathrm{mmHg}$ in the matched group, and the baseline BMI LSM was $36.57 \mathrm{~kg} / \mathrm{m}^{2}$ and $35.88 \mathrm{~kg} / \mathrm{m}^{2}$ in the COPE and in non-COPE, respectively (Table 3). The median number of HbA1c measurements preenrollment per subject was eight in both groups; the median number of post-enrollment measurements was seven for the COPE patients and eight for the nonCOPE patients [Table 3].

\section{Study outcomes}

Compared to mean adjusted $\mathrm{HbA} 1 \mathrm{c}$ in the 2 years prior to COPE enrollment, mean HbA1c in the two years after enrollment decreased by $-0.56 \%$ (95\% confidence interval (CI): $-0.69,-0.44)$ among COPE patients and increased by $+0.07 \%$ (95\%CI: $0.04,0.10)$ among the matched nonCOPE patients for a difference in differences of $0.63 \%$ (95CI\%: 0.50, 0.76) (Table 3).

This change in HbA1c among COPE versus non-COPE patients differed significantly in both non-adjusted and adjusted models. The adjusted change in LDL among COPE patients was $-10.58 \mathrm{mg} / \mathrm{dl},(95 \% \mathrm{CI}$ : $-15.85,-5.31)$ compared to $-3.18 \mathrm{mg} / \mathrm{dl}(95 \% \mathrm{CI}:-4.41,-1.95)$ among the non-COPE patients for a difference in differences of 7.40 (95\%CI: 2.00, 12.80). Systolic blood pressure increased in both groups, by $2.06 \mathrm{mmHg}(95 \% \mathrm{CI}: 1.10,3.02)$ in the intervention group and $0.61 \mathrm{mmHg}(95 \% \mathrm{CI}: 0.35,0.87)$ in the non-COPE group with a difference in differences of 1.45 (95\%CI: 0.46, 2.43). Changes in body mass index between the two groups were not significant $\left(-0.27 \mathrm{~kg} / \mathrm{m}^{2}\right.$ versus $-0.34 \mathrm{~kg} / \mathrm{m}^{2}$, with a difference in differences of 0.07, 95\%CI: - 0.34, 0.47). Results were consistent when we further adjusted the main models for a site-specific variable. In sensitivity analyses using one-year pre-post time windows, findings were consistent with the two-year results in terms of magnitude and direction for all the four clinical outcomes (Additional file 1 Table S1).

As shown in Tables 4, 48.92\% of the intervention group and $52.89 \%$ of the non-intervention group received "acceptable" disease monitoring between 12 and 24 months after enrollment. (adjusted odds ratio $(\mathrm{OR})=$ 0.93, 95\%CI: 0.68, 1.26) [Table 4].

\section{Discussion}

Participation in COPE among individuals living with T2DM was associated with improvements in HbA1c at 12 and 24 months and in LDL at 24 months. It is notable that changes in our cohort were sustained at 24 months, an outcome that is not usually assessed in evaluations of impact. The difference noted in HbA1c between the intervention and non-intervention groups over time reflects a clinically meaningful change which surpassed the average responses observed in T2DM self-management education programs described in a recent metaanalysis [14]. We also detected a significant reduction
Table 2 Baseline characteristics of COPE participants versus non-COPE participants, Navajo Nation, United States, 2010-2014

\begin{tabular}{llll}
\hline Characteristic & $\begin{array}{l}\text { COPE } \\
\mathrm{n}(\%)\end{array}$ & $\begin{array}{l}\text { non-COPE } \\
\mathrm{n}(\%)\end{array}$ & $\begin{array}{l}\mathrm{p}^{-} \\
\text {value }\end{array}$ \\
\hline $\begin{array}{l}\text { Total number of participants } \\
\text { Patient characteristics }\end{array}$ & 173 & 2880 & \\
Age & & & \\
$25-40$ y & $7(4.05)$ & $46(1.60)$ & 0.003 \\
$41-55$ y & $33(19.08)$ & $610(21.18)$ & \\
$56-70$ y & $74(42.77)$ & $1436(49.86)$ & \\
$71-85$ y & $54(31.21)$ & $764(26.53)$ & \\
$=>86$ y & $5(2.89)$ & $24(0.83)$ & \\
Gender & & & \\
Male & $65(37.57)$ & $901(31.28)$ & 0.084 \\
Female & $108(62.43)$ & $1979(68.72)$ & \\
Preferred Language & & & \\
Native American & $100(57.80)$ & $1178(40.90)$ & $<.0001$ \\
English & $72(41.62)$ & $1700(59.03)$ & \\
Missing & $1(0.58)$ & $2(0.07)$ & \\
Primary Care Physician & & & \\
Yes & $146(84.39)$ & $2577(89.48)$ & 0.036 \\
No & $27(15.61)$ & $303(10.52)$ & \\
\hline
\end{tabular}

Clinical Diagnoses

Essential Hypertension

Yes

113(65.32) $\quad$ 1966(68.26) $\quad 0.400$

No

$60(34.68)$

909(31.56)

Missing

$5(0.17)$

Major Depression Disorder

Yes

25(14.45)

282(9.79)

0.049

No

148(85.55)

2593(90.03)

Missing

$5(0.17)$

Alcohol abuse

Yes

11(6.36)

$77(2.67)$

0.015

No

162(93.64)

2798(97.15)

Missing

$5(0.17)$

Major Cardiovascular Disease

Yes

123(71.10)

2050(71.18)

0.954

No

50(28.90)

$825(28.65)$

Missing

$5(0.17)$

Dyslipidemia

$\begin{array}{llll}\text { Yes } & 80(46.24) & 1688(58.61) & 0.001\end{array}$

No 93(53.76) 1187(41.22)

Missing

$5(0.17)$

COPE Community Outreach and Patient Empowerment, y years, HbA1c glycosylated hemoglobin

Evaluation at the closest available $\mathrm{HbA} 1 \mathrm{c}$ measure before the enrollment date 
Table 3 Clinical outcomes comparisons at 24 months, Navajo Nation, United States, 2010-2014

\begin{tabular}{|c|c|c|c|c|c|c|}
\hline \multicolumn{3}{|c|}{ Intervention } & \multicolumn{2}{|l|}{ Crude Model $^{a}$} & \multicolumn{2}{|l|}{ Adjusted Model $^{\mathrm{b}}$} \\
\hline & & & Difference post VS pre intervention & $95 \% \mathrm{Cl}$ & Difference post VS pre intervention & $95 \% \mathrm{Cl}$ \\
\hline & PRE & POST & $\mathrm{HbA1c}$ & & & \\
\hline \multirow[t]{3}{*}{ COPE } & $N=173$ & $N=163$ & & & & \\
\hline & LSM (SE) & LSM (SE) & & & & \\
\hline & $8.39(0.05)$ & $7.82(0.06)$ & -0.57 & $-0.70,-0.45$ & -0.56 & $-0.69,-0.44$ \\
\hline \multirow[t]{5}{*}{ non-COPE } & $N=2879$ & $N=2738$ & & & & \\
\hline & LSM (SE) & LSM (SE) & & & & \\
\hline & $8.23(0.01)$ & $8.29(0.01)$ & 0.06 & $0.03,0.09$ & 0.07 & $0.04,0.10$ \\
\hline & & & $P$ interaction & $<0.0001$ & $P$ interaction & $<0.0001$ \\
\hline & PRE & POST & LDL & & & \\
\hline \multirow[t]{3}{*}{ COPE } & $N=72$ & $N=73$ & & & & \\
\hline & LSM (SE) & LSM (SE) & & & & \\
\hline & $95.70(3.85)$ & $83.92(3.87)$ & -11.79 & $-17.04,-6.53$ & -10.58 & $-15.85,-5.31$ \\
\hline \multirow[t]{5}{*}{ non-COPE } & $N=1596$ & $N=1499$ & & & & \\
\hline & LSM (SE) & LSM (SE) & & & & \\
\hline & $95.52(2.13)$ & $91.18(2.15)$ & -4.33 & $-5.54,-3.13$ & -3.18 & $-4.41,-1.95$ \\
\hline & & & $P$ interaction & 0.007 & $P$ interaction & 0.007 \\
\hline & PRE & POST & $\mathrm{SBP}$ & & & \\
\hline \multirow[t]{3}{*}{ COPE } & $N=113$ & $N=113$ & & & & \\
\hline & LSM (SE) & LSM (SE) & & & & \\
\hline & $132.46(0.97)$ & $134.86(0.97)$ & 2.40 & $1.45,3.35$ & 2.06 & $1.10,3.02$ \\
\hline \multirow[t]{5}{*}{ non-COPE } & $N=1966$ & $N=1964$ & & & & \\
\hline & LSM (SE) & LSM (SE) & & & & \\
\hline & $131.45(0.36)$ & $132.43(0.36)$ & 0.98 & $0.74,1.23$ & 0.61 & $0.35,0.87$ \\
\hline & & & $P$ interaction & 0.005 & $P$ interaction & 0.004 \\
\hline & PRE & POST & $\mathrm{BMI}$ & & & \\
\hline \multirow[t]{3}{*}{ COPE } & $N=59$ & $N=59$ & & & & \\
\hline & LSM (SE) & LSM (SE) & & & & \\
\hline & $36.57(0.71)$ & $36.06(0.71)$ & -0.51 & $-0.90,-0.12$ & -0.27 & $-0.67,0.12$ \\
\hline \multirow[t]{4}{*}{ non-COPE } & $N=1196$ & $N=1182$ & & & & \\
\hline & LSM (SE) & LSM (SE) & & & & \\
\hline & $35.88(0.16)$ & $35.31(0.16)$ & -0.57 & $-0.67,-0.47$ & -0.34 & $-0.45,-0.22$ \\
\hline & & & $P$ interaction & 0.78 & $P$ interaction & 0.75 \\
\hline
\end{tabular}

COPE Community Outreach and Patient Empowerment, HbA1c glycosylated hemoglobin, LDL low-density lipoprotein ( $\mathrm{mg} / \mathrm{dl}), S B P$ systolic blood pressure (mmHg), $B M I$ body mass index $\left(\mathrm{kg} / \mathrm{m}^{2}\right)$, LSM Least Squares Means, SE Standard Error, Cl confidence interval, $P$ interaction $\mathrm{p}$-value of the interaction for the $\beta$ of the term intervention $(0 / 1)^{*}$ time (pre/post)

${ }^{\text {a }}$ Model adjusted for the pre-2 years' means

b Adjusted model for pre-2 years' means, age (years; continuous), gender (male/female), preferred language (English, Navajo and other), primary care physician (yes/no); essential hypertension (yes/no), major depression disorder (yes/no), alcohol abuse (yes/no), dyslipidemia (yes/no), and major cardiovascular disease (yes/no)

in LDL among COPE patients compared to non-COPE patients who had a baseline diagnosis of dyslipidemia. We observed a slight increase in blood pressure for both COPE and non-COPE patients. Lastly, we did not see improved changes in body mass index, or monitoring of standard clinical measures related to cardiovascular risk due to COPE participation.
Our results are consistent with those observed in the most effective community-based DM interventions reported in a systematic review of nutrition-based interventions among American Indian and other indigenous populations [3]. Our observed decline of HbA1c ($0.42 \%$ versus $+0.04 \%$ and $-0.56 \%$ versus $+0.07 \%$ respectively at 1 and 2 years) was similar to the mean 
Table 4 Logistic models for "disease monitoring" outcome, Navajo Nation, United States, 2010-2014

\begin{tabular}{llll}
\hline Rate of received "acceptable" disease monitoring & Intervention & OR & \multicolumn{1}{c}{$95 \% \mathrm{Cl}$} \\
\hline $48.92 \%$ in COPE & COPE vs non-COPE ${ }^{a}$ & 0.85 & $0.63,1.15$ \\
$52.89 \%$ in non-COPE & COPE vs non-COPE ${ }^{\text {b }}$ & 0.93 & $0.68,1.26$ \\
\hline
\end{tabular}

COPE Community Outreach and Patient Empowerment, OR Odds ratio, $\mathrm{Cl}$ confidence interval

a Model adjusted for baseline monitoring disease (indicator variable that takes value 1 if $\mathrm{HbA1C}$, LDL, and SBP, are all measured at least once in the 12 months before the enrollment date, and 0 otherwise)

${ }^{\mathrm{b}}$ Adjusted model for baseline monitoring disease, age (years; continuous), gender (male/female), preferred language (English, Navajo and other), primary care physician (yes/no); essential hypertension (yes/no), major depression disorder (yes/no), alcohol abuse (yes/no), dyslipidemia (yes/no), and major cardiovascular disease (yes/no)

Models evaluate the probability of having glycosylated hemoglobin (HbA1c), low-density lipoprotein (LDL), and systolic blood pressure (SBP), all been measured at least once between 12 and 24 months after the enrollment date

HbA1c changes in three studies that compared people receiving versus not receiving the intervention (ranging from $+0.38 \%$ versus -0.46 , $+0.4 \%$ versus $+1.2 \%$, to $0.8 \%$ versus $+0.3 \%)[15-17]$. The American Diabetes Association cites $0.5 \% \mathrm{HbA} 1 \mathrm{c}$ as a clinically significant change [18]. For context, a $0.5 \%$ increase in HbA1c is associated with $9 \%$ higher odds of myocardial infarction [19], and an HbA1c reduction from 8.3 to $8.0 \%$ has been shown to significantly reduce the risk of retinopathy over an approximate 10-year span [20]. Furthermore, for our population of predominantly elder individuals, the average $\mathrm{HbA1c}$ achieved of 7.82 at 24 months likely reflect optimal control based on recommendations by the American College of Physicians [21]. The reduction of LDL by $-10.58 \mathrm{mg} / \mathrm{dl}$ observed in this study is comparable to decline in LDL levels of $-5.29 \mathrm{mg} / \mathrm{dl}$ noted by Moore et al. in a large-scale, intensive intervention among American Indian and Alaska Native populations with T2DM [22]. The 12\% drop in LDL that we observed in our cohort confers meaningful clinical benefit, including a $19 \%$ relative reduction in the risk of nonfatal myocardial infarction or death due to coronary health disease $[23,24]$. In contrast to these results, both COPE and non-COPE participants experienced a modest increase in SBP during the 2-year follow-up, with a small but statistically significant increase among COPE patients $(+2.06$ versus $+0.61 \mathrm{mmHg})$. We believe this increase has minimal clinical significance, considering the mean changes observed in clinical trials aimed at improving hypertension through $\mathrm{CHW}$ interventions, which ranged from $-6.5 \mathrm{mmHg}$ versus $-2.7 \mathrm{mmHg}$, $-7.5 \mathrm{mmHg}$ versus $+3.4 \mathrm{mmHg}$, to $-10.8 \mathrm{mmHg}$ versus $-5.8 \mathrm{mmHg}$ among people receiving versus people not receiving the intervention [25-27]. Neither COPE nor non-COPE participants experienced improvements in BMI.

Our findings are consistent with the growing evidence that supports the integration of community health workers (CHWs) on clinical care team, especially in underserved groups (African-American, Hispanic, or groups classified as low-income) [28]. Other studies have documented a significant impact of CHW-based interventions on non-communicable chronic diseases in the US, including improvements in cholesterol and blood pressure [29], as well as in reducing costs and preventable healthcare utilization [30]. A recent systematic review of $\mathrm{CHW}$ intervention for DM found a range of improved outputs and outcomes including knowledge about DM, diabetes self-care behaviors, clinical outcomes, and healthcare utilization and costs especially in Hispanic and non-Hispanic Black populations [31].

We note some limitations of our study. Firstly, while the use of routine clinical data provided a unique opportunity to compare COPE participants with thousands of individuals with comparable clinical indicators, not all the clinical outcomes were consistently measured and other variables related to sociodemographic characteristics and patient behavior were unavailable and could not be assessed as potential confounders or mediators. However we speculate that even if sociodemographic and behavioral differences exist between COPE and non-COPE patients, they would be likely skewed toward greater vulnerability among the intervention group. Second, this study was limited to individuals living with T2DM who were seen at a participating facility. Thus, findings may not be representative of DM patients who were not engaged in healthcare at all or seen at other facilities. We also note that we could have underestimated the impact of our intervention if there were positive spillovers effects within our sites, since the same CHRs also see non-COPE clients in addition to those enrolled in COPE. However, we estimate that the nonCOPE patients seen by CHRs would only represent a small proportion of the enter non-intervention group $(<5 \%)$, unlike case of "study contamination" would likely bias toward the null.

\section{Conclusion}

In a programmatic intervention carried out in a Native community, we observed improved diabetes outcomes, sustained over two years, among patients receiving structured $\mathrm{CHW}$ outreach. Our program findings support the growing evidence that $\mathrm{CHW}$ are effective in reducing chronic disease disparities, particularly when, 
CHWs are an integrated part of the healthcare. Given the prevalence of diabetes among underserved populations and the literature to support the role of community health workers in reducing health disparities [32], the findings of this study provide further evidence to support expansion of the CHW workforce as uniquely valuable healthcare professionals and widespread integration of CHW in U.S. healthcare systems. Future work will focus on understanding health utilization patterns of COPE patients, understanding the impact of COPE on patient-reported outcomes, and identifying patient characteristics that predict treatment response.

\section{Supplementary information}

Supplementary information accompanies this paper at https://doi.org/10. 1186/s12939-019-1097-9.

Additional file 1: Table S1. and Appendix. Table S1 reports the results for clinical outcomes comparisons at 12 months; the Appendix reports the details of the linear mixed models.

\section{Abbreviations}

Cl: confidence interval; LSM: Least Square Mean; OR: odds ratio

\section{Acknowledgements}

We would like to thank the Patients-Centered Outcomes Research Institute (PCORI), the patients, the CHRs, the Navajo Nation, our community health advisory groups, the clinical providers, and the data collection specialists. We are very grateful to the Community Health Advisory Panel (CHAP) and COPE Advisory Group (CAG). In particular, we would like to acknowledge the patients and CHRs of CHAP including Bobby Talk, Judy Singer, Alfred Kahn, William Mosberger, Norbert Yazzie, Stephanie Benally, Lenora Shirley, and Cornelia Keams. Without their valuable contribution our research would no have been possible. In addition, we are grateful to Professor Megan Murray for her helpful input and comments. The opinions expressed in this paper are those of the author(s) and do not necessarily reflect the views of the IHS.

\section{Competing interests}

Dr. Sonya S. Shin is the Executive Director of a 501(c)3 called Community Outreach and Patient Empowerment, which has continued to provide the services described in this study. The other authors declare that they have no conflict of interest to disclose.

\section{Authors' contributions}

LT coded, analyzed data and drafted the manuscript. JEO supported the statistical methodology and helped to revise the manuscript. SA cleaned the raw data, pre-prepared the data for the analysis, helped with the statistical methodology. CB and KAN helped design the study. CK, OM, CB, and HS, helped to coordinate the project and related activities. CC extracted the data from the RPMS. CK, OM, HS, MGB contributed in the revision of the manuscript. SSS designed the study, provided clinical insights, helped to draft the manuscript. All authors contributed in revising the manuscript and have given final approval of the version to be published. SSS is the guarantor of this work and, as such, had full access to all the data in the study and takes responsibility for the integrity of the data and the accuracy of the data analysis.

\section{Funding}

Research reported in this publication was funded through a Patient-Centered Outcomes Research Institute (PCORI) Award (AD-1304-6566). The statements in this publication are solely the responsibility of the authors and do not necessarily represent the views of the Patient-Centered Outcomes Research Institute (PCORI), its Board of Governors or Methodology Committee.

\section{Availability of data and materials}

The datasets generated and/or analyzed during the current study are not publicly available due to Navajo Human Research Review Board (NHRRB) policy that all research data collected in Navajo Nation belong to the Navajo Nation; they are available from the NHRRB on reasonable request.

\section{Ethics approval and consent to participate}

This study was approved by Partners Healthcare Institutional Review Board (2012P001069) and the Navajo Nation Human Research Review Board (NNR11.150 T). Patients provided verbal consent to participate in the programmatic intervention COPE.

\section{Consent for publication}

Not applicable.

\section{Author details}

'Department of Global Health and Social Medicine, Harvard Medical School, Boston, MA, USA. Department of Medicine, Brigham and Women's Hospital, Boston, MA, USA. 'Division of Global Health Equity, Brigham and Women's Hospital, Boston, MA, USA. ${ }^{4}$ School of Medicine, Oregon Health and Science University, Portland, OR, USA. ${ }^{5}$ Partners in Health, Boston, MA, USA. ${ }^{6}$ Navajo Department of Health, Window Rock, Window Rock, AZ, USA.

Received: 3 July 2019 Accepted: 18 November 2019 Published online: 27 November 2019

\section{References}

1. Navajo Department of Health. Navajo epidemiology center update. 2016. https://www.nec.navajo-nsn.gov/Portals/0/Announcements/Navajo\%2 OEpidemiology\%20Center\%20Update\%20May\%202016.pdf . Published April 15, 2016.

2. Indian Health Service, Department of Health and Human Services. Trends in Indian Health 2014 edition. https://www.nihb.org/tribalhealthreform/wpcontent/uploads/2015/10/IHS-Trends-2014-Book-508.pdf

3. Nava LT, Zambrano JM, Arviso KP, et al. Nutrition-based interventions to address metabolic syndrome in the Navajo: a systematic review. J Clin Nurs. 2015;24(21-22):3024-45

4. Liao Y, Bang D, Cosgrove S, et al. Surveillance of health status in minority communities - racial and ethnic approaches to community health across the U.S. (REACH U.S.) risk factor survey, United States, 2009. MMWR Surveill Summ Morb Mortal Wkly report / CDC. 2011;60(6):1-44.

5. Dine' Policy Institute. Dine' food sovereignty report.; 2014. https://www. dinecollege.edu/wp-content/uploads/2018/04/dpi-food-sovereignty-report. pdf. Published April 28, 2018

6. Indian Health Service. https://www.ihs.gov/chr/. Published March 16, 2018.

7. Howard BV, Robbins DC, Sievers ML, et al. LDL cholesterol as a strong predictor of coronary heart disease in diabetic individuals with insulin resistance and low LDL: the strong heart study. Arterioscler Thromb Vasc Biol. 2000:20:830-5.

8. Lu WQ, Resnick HE, Jablonski KA, et al. Effects of glycaemic control on cardiovascular disease in diabetic American Indians: the strong heart study. Diabet Med. 2004;21(4):311-7.

9. Bureau USC. 2011. https://www.census.gov/newsroom/releases/archives/ facts_for_features_special_editions/cb11-ff22.html. Published November 1, 2011

10. Council. HDNANN. Healthy Dine' Nations Act. Navajo Nation Council. 2014 http://www.navajo-nsn.gov/News\%20Releases/OPVP/2014/nov/Healthy\%2 0Dine\%20Nation\%20Act\%20of\%202014.pdf. Published November 21, 2014

11. King C, Goldman A, Gampa V, et al. Strengthening the role of community health representatives in the Navajo Nation. BMC Public Health. 2017;17(1): $1-10$

12. Wadden TA, Butryn ML, Hong PS, et al. Behavioral treatment of obesity in patients encountered in primary care settings: a systematic review. Jama. 2014;312(17):1779-91.

13. Grandes G, Sanchez A, Cortada JM, et al. Is integration of healthy lifestyle promotion into primary care feasible? Discussion and consensus sessions between clinicians and researchers. BMC Health Serv Res. 2008;8:1-12.

14. Chrvala CA, Sherr D, Lipman RD. Diabetes self-management education for adults with type 2 diabetes mellitus: a systematic review of the effect on glycemic control. Patient Educ Couns. 2016;99(6):926-43. 
15. Daniel M, Green LW, Marion SA, et al. Effectiveness of community-directed diabetes prevention and control in a rural aboriginal population in British Columbia, Canada. Soc Sci Med. 1999;48(6):815-32.

16. Gilliland SS, Azen SP, Perez GE, et al. Strong in body and spirit lifestyle intervention for native american adults with diabetes in New Mexico. Diabetes Care. 2002;25(1):78-83.

17. Robertson C, Kattelmann K, Ren C. Control of type 2 diabetes mellitus using interactive internet-based support on a Northern Plains Indian Reservation: a pilot study. Top Clin Nutr. 2007;22(2):185-93.

18. American Diabetes Association. Standards of medical care in diabetes 2009. Diabetes Care. 2009;32(Suppl 1):S13-61.

19. Gerstein $\mathrm{HC}$, Islam S, Anand S, et al. Dysglycaemia and the risk of acute myocardial infarction in multiple ethnic groups: an analysis of 15,780 patients from the INTERHEART study. Diabetologia. 2010;53(12):2509-17.

20. Lind $M$, Odén $A$, Fahlén $M$, et al. The shape of the metabolic memory of $\mathrm{HbA1C}$ : re-analysing the DCCT with respect to time-dependent effects. Diabetologia. 2010;53(6):1093-8.

21. Qaseem A, Wilt TJ, Kansagara D, et al. Hemoglobin A1c targets for glycemic control with pharmacologic therapy for nonpregnant adults with type 2 diabetes mellitus: a guidance statement update from the American college of physicians. Ann Intern Med. 2018;168(8):569-76.

22. Moore K, Jiang L, Manson SM, et al. Case management to reduce cardiovascular disease risk in American Indians and Alaska natives with diabetes: results from the special diabetes program for Indians healthy heart demonstration project. Am J Public Health. 2014;104(11):e158-64.

23. Program $L R C$. The lipid research clinics coronary primary prevention trial results: I. reduction in incidence of coronary heart disease. JAMA. 1984; 251(3):351-64.

24. Mihaylova B, Emberson J, Blackwell L, et al. The effects of lowering LDL cholesterol with statin therapy in people at low risk of vascular disease: meta-analysis of individual data from 27 randomised trials. Lancet. 2012; 380(9841):581-90.

25. Levin DM, Bone LR, Hill MN, et al. The effectiveness of a community/academic health center partnership in decreasing the level of blood pressure in an urban African-American population. Ethn Dis. 2003;13(3):354-61.

26. Hill MN, Han HR, Dennison CR, et al. Hypertension care and control in underserved urban African American men: behavioral and physiologic outcomes at 36 months. Am J Hypertens. 2003;16(11):906-13.

27. Jafar $\mathrm{TH}$, Hatcher J, Poulter $\mathrm{N}$, et al. Community-based interventions to promote blood pressure control in a developing country: a cluster randomized trial. Ann Intern Med. 2009;151(9):593-601.

28. Centers of Disease Control and Prevention. Integrating community health workers on clinical care teams and in the community. https:/wwww.cdc.gov/ dhdsp/pubs/docs/Best_Practice_Guide_CHW_508.pdf. Published January 28, 2018.

29. Kim K, Choi JS, Choi E, et al. Effects of community-based health worker interventions to improve chronic disease management and care among vulnerable populations: a systematic review. Am J Public Health. 2016; 106(4):e3-e28.

30. Jack HE, Arabadjis SD, Sun L, et al. Impact of community health workers on use of healthcare Services in the United States: a systematic review. J Gen Intern Med. 2017;32(3):325-44.

31. Golden SH, Maruthur N, Mathioudakis N, et al. The case for diabetes population health improvement: evidence-based programming for population outcomes in diabetes. Curr Diab Rep. 2017;17(7):51.

32. Balcazar H, Rosenthal EL, Brownstein JN, et al. Community health workers can be a public health force for change in the United States: three actions for a new paradigm. Am J Public Health. 2011;101(12):2199-203.

\section{Publisher's Note}

Springer Nature remains neutral with regard to jurisdictional claims in published maps and institutional affiliations.

Ready to submit your research? Choose BMC and benefit from:

- fast, convenient online submission

- thorough peer review by experienced researchers in your field

- rapid publication on acceptance

- support for research data, including large and complex data types

- gold Open Access which fosters wider collaboration and increased citations

- maximum visibility for your research: over $100 \mathrm{M}$ website views per year

At BMC, research is always in progress.

Learn more biomedcentral.com/submissions 